\title{
Torsional Rigidity of a Circular Bar with Multiple Circular Inclusions using a Null-Field Integral Approach
}

\author{
Ying-Te Lee*, Jeng-Tzong Chen, An-Chien Wu \\ Department of Harbor and River Engineering, National Taiwan Ocean University, Keelung 20224, \\ Taiwan, China
}

Email: D93520002@mail.ntou.edu.tw,jtchen@mail.ntou.edu.tw, M93520008@mail.ntou.edu.tw

\begin{abstract}
In this paper, a systematic approach is proposed to calculate the torsional rigidity of a circular bar containing multiple circular inclusions. To fully capture the circular geometries, the kernel function is expanded to the degenerate form and the boundary density is expressed into Fourier series. The approach is seen as a semi-analytical manner since error purely attributes to the truncation of Fourier series. By collocating the null-field point exactly on the real boundary and matching the boundary condition, a linear algebraic system is obtained. After obtaining the unknown Fourier coefficients, the solution can be obtained by using the integral representation. Finally, torsion problems are revisited to check the validity of our method. Torsional rigidities for a circular bar with an eccentric inclusion are compared well with the exact solution, BEM data and the Tang's results. Convergence study shows that only a few number of Fourier series terms can yield acceptable results. The torsional rigidities of two limiting case of cavity and rigid inclusion are also obtained using the present approach. Five gains of well-posed model, singularity free, free of boundary-layer effect, exponential convergence and mesh-free approach are achieved. A general-purpose program was developed to determine the torsional rigidity for a circular bar with arbitrary number, radii, positions and shear moduli of circular inclusions.
\end{abstract}

Keywords: Torsional rigidity, Null-field integral equation, inclusion

\section{INTRODUCTION}

In the past, multiply-connected problems have been solved either by conformal mapping or by other techniques. Ling [1] solved the torsion problem of a circular bar with several holes. Muskhelishvili [2] solved the problem of a circular bar reinforced by an eccentric circular inclusion. Chen and Weng [3] have introduced conformal mapping with a Laurent series expansion to analyze the Saint-Venant torsion problem. They concerned with an eccentric bar of different materials with an imperfect interface under torque. Since the conformal mapping is limited to the doubly-connected region, it encounters difficulty for multiple inclusions. Therefore, many researchers have paid more attentions on other techniques or numerical methods. In 1983, Caulk [4] developed a special boundary integral method to deal with the problem of a torsion bar with circular holes. Shams-Ahmadi and Chou [5] used the complex variable boundary element method (CVBEM) to solve the torsion problem of composite shafts with any number of inclusions of different materials. Ang and Kang [6] developed a general formulation for solving the second-order elliptic partial differential equation for a multiply-connected region in a different version of CVBEM. Petrov [7] developed an effective technique of boundary element method (BEM) to determine torsion, shear and other characteristics of beam cross-sections of arbitrary complex shape including multiply-connected cross sections. Tang [8] utilized the singular and hypersigular formulations to solve the torsion problem with inclusions and/or cracks. It may be easy to deal with two-dimensional problems. For 
three-dimensional problems, it may become more complex since the boundary surface needs to be discretized. Recently, meshless methods $[9,10]$ become very popular, since it is free of mesh generation and only nodes are needed. The present formulation can be seen as one kind of meshless methods, since it belongs to the boundary collocation method. Mogilevskaya and Crouch [11] have solved the problem of an infinite plane containing arbitrary number of circular inclusions based on the complex singular integral equation. Later, they [12] utilized Somigliana's formula and Fourier series for elasticity problems with circular boundaries. In their analysis procedure, the unknown tractions are approximated by using the complex Fourier series. However, for calculating an integral over a circular boundary, they didn't expand the fundamental solution to degenerate kernel using the polar coordinate of local system. By moving the null-field point exactly on the real boundary, the boundary integral can be easily determined using series sums in our formulation due to the introduction of degenerate kernels. Mogilevskaya and Crouch [11] have used the Galerkin method instead of collocation approach. Free of worrying how to choose the collocation points, uniform collocation along the circular boundary yields a well-conditioned matrix. On the other hand, Bird and Steele [13] have also used separated solution procedure for bending of circular plates with circular holes in a similar way of the Trefftz method by using the addition theorem. Recently, Chen and his coworkers [14] have utilized the null-field integral equations in conjunction with the degenerate kernel and Fourier series to solve the torsion problem of a circular bar including multiple circular holes. Following the success of [14], we extend to solve torsion problems with multiple circular inclusions.

In this paper, the null-field integral equation is utilized to solve the Saint-Venant torsion problem of a circular bar with circular inclusions. The mathematical tools, the degenerate kernel for the fundamental solution and Fourier series for the boundary density, are utilized in the null-field integral formulation. By collocating the null-field point exactly on the real boundary and matching the boundary condition, the linear algebraic system is obtained and the unknown Fourier coefficients can be easily determined. Then, series solutions for the warping function and torsional rigidity are obtained. Numerical examples are given to show the validity and efficiency of our formulation.

\section{FORMULATION OF THE PROBLEM}

A circular bar containing $N$ circular inclusions bounded to the contours $B_{k}(k=0,1,2, \cdots, N)$ is shown in Figure 1.

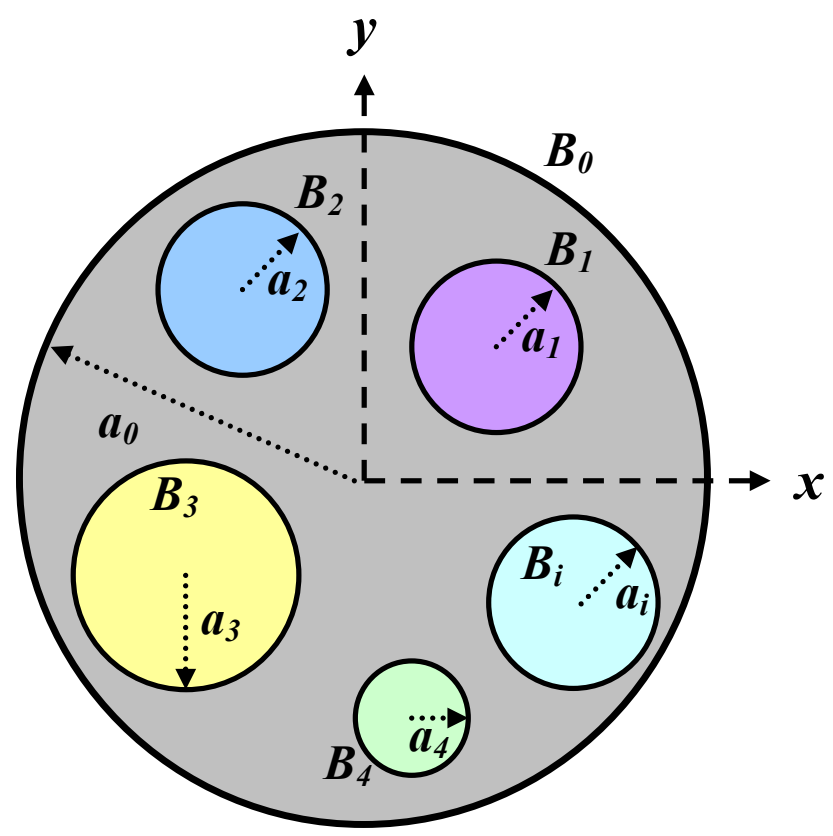

Figure 1: Sketch of a circular bar with circular inclusions and/or holes under torsion

We define

$B=\bigcup_{k=0}^{N} B_{k}$ 
The radii of the outer circle and the inner inclusions are $a_{0}$ and $a_{i}(i=1,2, \cdots, N)$, respectively. The circular bar twisted by couples applied at the end is taken into consideration. Following the theory of Saint-Venant torsion [15], we assume the displacement field to be

$u=-\alpha y z, v=\alpha x z, w=\alpha \varphi(x, y)$,

where $\alpha$ is the angle of twist per unit length along the $z$ direction and $\varphi$ is the warping function. According to the displacement field in Eq.(2), the strain components are

$\varepsilon_{x}=\varepsilon_{y}=\varepsilon_{z}=\gamma_{x y}=0$,

$\gamma_{x z}=\frac{\partial w}{\partial x}+\frac{\partial u}{\partial z}=\alpha\left(\frac{\partial w}{\partial x}-y\right)$

$\gamma_{y z}=\frac{\partial w}{\partial y}+\frac{\partial v}{\partial z}=\alpha\left(\frac{\partial w}{\partial y}+x\right)$

and their corresponding components of stress are

$\sigma_{x}=\sigma_{y}=\sigma_{z}=\sigma_{x y}=0$,

$\sigma_{x z}=\mu \alpha\left(\frac{\partial w}{\partial x}-y\right), \quad \sigma_{y z}=\mu \alpha\left(\frac{\partial w}{\partial y}+x\right)$,

where $\mu$ is the shear modulus. There is no distortion in the planes of cross sections since $\varepsilon_{x}=\varepsilon_{y}=\varepsilon_{z}=\gamma_{x y}=0$. We have the state of pure shear at each point defined by the stress components $\sigma_{x z}$ and $\sigma_{y z}$. The warping function $\varphi$ must satisfy the equilibrium equation

$\frac{\partial^{2} \varphi}{\partial x^{2}}+\frac{\partial^{2} \varphi}{\partial y^{2}}=0$ in $D$,

where the body force is neglected and $D$ is the domain. Since there are no external forces on the cylindrical surface, we have free traction, $t_{x}=t_{y}=t_{z}=0$. By substituting the normal vector, the only zero traction $t_{z}$ becomes

$t_{z}=\sigma_{x z} n_{x}+\sigma_{y z} n_{y}=0$.

By substituting (7) into (9), the boundary condition is

$\frac{\partial \varphi}{\partial x} n_{x}+\frac{\partial \varphi}{\partial y} n_{y}=y n_{x}-x n_{y}=\nabla \varphi \cdot \mathbf{n}=\frac{\partial \varphi}{\partial n}$.

Since the traction-free condition is specified for the outer boundary, we have

$\frac{\partial \varphi}{\partial n}=0$ on $B_{0}$.

The continuity condition for the displacement and equilibrium condition for traction on the interface between the matrix and inclusion, are

$\varphi^{M}=\varphi^{I}$ on $B_{i}$,

$\mu_{0} \frac{\partial \varphi_{i}^{M}}{\partial n}-\mu_{i} \frac{\partial \varphi_{i}^{I}}{\partial y}=\left(\mu_{0}-\mu_{i}\right)\left(y n_{x}-x n_{y}\right)$ on $B_{i}$,

where the superscripts " $P$ " and " $M$ " denote inclusion and matrix, respectively, $\mu_{0}$ is the shear modulus for the matrix and $\mu_{i}$ is the shear modulus for the $i t h$ inclusion. 
1. Dual boundary integral equations and dual null-field integral equations The integral equation for the domain point can be derived from the third Green's identity [16], we have

$$
\begin{aligned}
& 2 \pi \varphi(x)=\int_{B} T(s, x) \varphi(s) d B(s)-\int_{B} U(s, x) \psi(s) d B(s), x \in D, \\
& 2 \pi \frac{\partial \varphi(x)}{\partial n_{x}}=\int_{B} M(s, x) \varphi(s) d B(s)-\int_{B} L(s, x) \psi(s) d B(s), \quad x \in D,
\end{aligned}
$$

where $s$ and $x$ are the source and field points, respectively, $D$ is the domain of interest, $\psi(s)=\frac{\partial \varphi(s)}{\partial n_{s}}, n_{s}$ and $n_{x}$ denote the outward normal vectors at the source point $s$ and field point $x$, respectively, and the kernel function $U(s, x)=\ln r,(r \equiv|s-x|)$, is the fundamental solution which satisfies

$$
\nabla^{2} U(s, x)=2 \pi \delta(x-s)
$$

in which $\delta(x-s)$ denotes the Dirac-delta function. The other kernel functions, $T(s, x), L(s, x)$, and $M(s, x)$, are defined by

$$
T(s, x)=\frac{\partial U(s, x)}{\partial n_{s}}, L(s, x)=\frac{\partial U(s, x)}{\partial n_{x}}, M(s, x)=\frac{\partial^{2} U(s, x)}{\partial n_{s} \partial n_{x}}
$$

By moving the field point to the boundary, Eqs.(14) and (15) reduce to

$$
\begin{aligned}
& \pi \varphi(x)=C . P . V . \int_{B} T(s, x) \varphi(s) d B(s)-R . P . V . \int_{B} U(s, x) \psi(s) d B(s), x \in B, \\
& \pi \frac{\partial \varphi(x)}{\partial n_{x}}=H . P . V . \int_{B} M(s, x) \varphi(s) d B(s)-C . P . V \cdot \int_{B} L(s, x) \psi(s) d B(s), x \in B,
\end{aligned}
$$

where R.P.V., C.P.V. and H.P.V. denote the Riemann principal value, Cauchy principal value and Hadamard principal value, respectively. Once the field point $x$ locates outside the domain $\left(x \in D^{c}\right)$, we obtain the dual null-field integral equations as shown below

$$
\begin{aligned}
& 0=\int_{B} T(s, x) \varphi(s) d B(s)-\int_{B} U(s, x) \psi(s) d B(s), \quad x \in D^{c}, \\
& 0=\int_{B} M(s, x) \varphi(s) d B(s)-\int_{B} L(s, x) \psi(s) d B(s), \quad x \in D^{c},
\end{aligned}
$$

where $D^{c}$ is the complementary domain. Eqs.(14), (15), (20) and (21) are conventional formulations where the point can not be located on the real boundary. Singularity occurs and concept of principal values is required once Eqs.(18) and (19) are considered. By introducing the degenerate kernel, we can located the point exactly on the real boundary for Eqs.(20) and (21) as shown below:

2. Expansions of fundamental solution and boundary density Based on the separable property, the kernel function $U(s, x)$ can be expanded into degenerate form by separating the source points and field points in the polar coordinate [17]:

$$
U(s, x)= \begin{cases}U^{i}(R, \theta ; \rho, \phi)=\ln R-\sum_{m=1}^{\infty} \frac{1}{m}\left(\frac{\rho}{R}\right)^{m} \cos m(\theta-\phi), & R \geq \rho, \\ U^{e}(R, \theta ; \rho, \phi)=\ln \rho-\sum_{m=1}^{\infty} \frac{1}{m}\left(\frac{R}{\rho}\right)^{m} \cos m(\theta-\phi), & R<\rho,\end{cases}
$$

where the superscripts " $i$ " and " $e$ " denote the interior $(R>\rho)$ and exterior $(R<\rho)$ cases, respectively. In order to ensure the log singularity and the series convergence, the leading term and the numerator in the above expansion is dominated by the larger argument. After taking the derivative operators in Eq.(17), the $T(s, x), L(s, x)$ and $M(s, x)$ kernels can be easily derived and the detailed representation can be found in [14]. It is noted that the null-field point in Eq.(20) or the domain point in Eq.(14) can be exactly located on the 
real boundary when the appropriate degenerate kernels are employed. Our formulation for Eqs.(14), (15), (20) and (21) can be used for the point $x$ on the real boundary free of singular integrals, while the conventional BEM needs to deal with singularities.

For the boundary densities, we apply the Fourier series expansions to approximate the potential $\varphi$ and its normal derivative $\psi$ on the boundary

$$
\begin{aligned}
& \varphi\left(s_{k}\right)=a_{0}^{k}+\sum_{n=1}^{\infty}\left(a_{n}^{k} \cos n \theta+b_{n}^{k} \sin n \theta\right), \quad s_{k} \in B_{k}, \quad k=1,2, \cdots, N, \\
& \psi\left(s_{k}\right)=p_{0}^{k}+\sum_{n=1}^{\infty}\left(p_{n}^{k} \cos n \theta+q_{n}^{k} \sin n \theta\right), \quad s_{k} \in B_{k}, \quad k=1,2, \cdots, N,
\end{aligned}
$$

where $a_{n}^{k}, b_{n}^{k}, p_{n}^{k}$ and $q_{n}^{k}$ are the Fourier coefficients and $\theta$ is the polar angle $(0<\theta<2 \pi)$.

3. Adaptive observer system After moving the point of Eq. (20) to the boundary, the boundary integrals through all the circular contours are required. Since the boundary integral equations are frame indifferent, i.e. objectivity rule is satisfied. The observer system is adaptively to locate the origin at the center of each circle in the boundary integrals. Adaptive observer system is chosen to fully employ the property of degenerate kernels. Figures 2(a) and 2(b) show the boundary integration for the circular boundaries in the adaptive observer system. It is noted that the origin of the observer system is located on the center of the corresponding circle under integration to entirely utilize the geometry of circular boundary for the expansion of degenerate kernels and boundary densities. The dummy variable in the circular integration is angle $(\theta)$ instead of radial coordinate $(R)$.

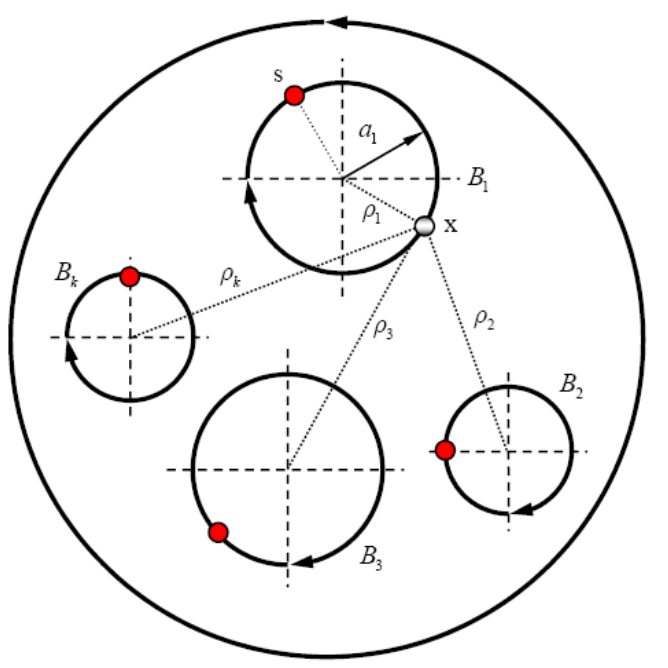

Figure 2(a): Sketch of the null-field integral equation in conjunction with the adaptive observer system

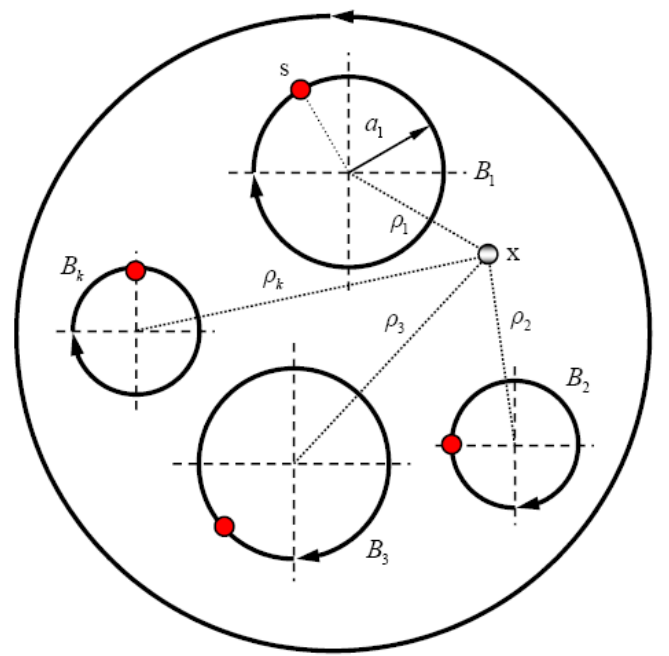

Figure 2(b): Sketch of the boundary integral equation for the domain point in conjunction with the adaptive observer system

4. Linear algebraic system By moving the null-field point $x_{k}$ to exactly locate on the $k$ th circular boundary in the sense of limit for Eq. (20) in Figure 2(a), we have

$0=\sum_{k=0}^{N} \int_{B_{k}} T(s, x) \varphi(s) d B_{k}(s)-\sum_{k=0}^{N} \int_{B_{k}} U(s, x) \psi(s) d B_{k}(s), \quad x \in D^{c}$,

where $N$ is the number of circles including the outer boundary and the inner circular holes. In the real computation, we select the collocation point on the boundary. It is noted that the integration path is counterclockwise for the outer circle. Otherwise, it is clockwise. For the $B$ integral of the circular boundary, the kernels of $U(s, x)$ and $T(s, x)$ are expressed in terms of degenerate kernels, and $\varphi(s)$ and $\psi(s)$ are substituted by using the Fourier series. In the $B_{k}$ integral, we set the origin of the observer system to collocate at the center $c_{k}$ to fully utilize the degenerate kernels and Fourier series. By collocating the null-field point exactly on the boundary, a linear algebraic system is obtained 
$[\mathbf{U}]\{\boldsymbol{\psi}\}=[\mathbf{T}]\{\varphi\}$,

where $[\mathbf{U}]$ and $[\mathbf{T}]$ are the influence matrices with a dimension of $N \times(2 L+1)$ by $N \times(2 L+1),\{\varphi\}$ and $\{\psi\}$ denote the column vectors of Fourier coefficients with a dimension of $N \times(2 L+1)$ by 1 in which $[\mathbf{U}],[\mathbf{T}],\{\varphi\}$ and $\{\boldsymbol{\psi}\}$ can be defined as follows:

$$
\begin{aligned}
& {[\mathbf{U}]=\left[\begin{array}{cccc}
\mathbf{U}_{00} & \mathbf{U}_{01} & \cdots & \mathbf{U}_{0 N} \\
\mathbf{U}_{10} & \mathbf{U}_{11} & \cdots & \mathbf{U}_{1 N} \\
\vdots & \vdots & \ddots & \vdots \\
\mathbf{U}_{N 0} & \mathbf{U}_{N 0} & \cdots & \mathbf{U}_{N N}
\end{array}\right], \quad[\mathbf{T}]=\left[\begin{array}{cccc}
\mathbf{T}_{00} & \mathbf{T}_{01} & \cdots & \mathbf{T}_{0 N} \\
\mathbf{T}_{10} & \mathbf{T}_{N N} & \cdots & \mathbf{T}_{1 N} \\
\vdots & \vdots & \ddots & \vdots \\
\mathbf{T}_{N 0} & \mathbf{T}_{N 1} & \cdots & \mathbf{T}_{N N}
\end{array}\right],} \\
& \{\varphi\}=\left\{\begin{array}{c}
\varphi_{0} \\
\varphi_{1} \\
\varphi_{2} \\
\vdots \\
\varphi_{N}
\end{array}\right\},\{\boldsymbol{\psi}\}=\left\{\begin{array}{c}
\boldsymbol{\Psi}_{0} \\
\boldsymbol{\Psi}_{1} \\
\boldsymbol{\Psi}_{2} \\
\vdots \\
\boldsymbol{\Psi}_{N}
\end{array}\right\} .
\end{aligned}
$$

where the vectors $\left\{\varphi_{k}\right\}$ and $\left\{\boldsymbol{\psi}_{k}\right\}$ are in the form of $\left\{a_{0}^{k} a_{1}^{k} b_{1}^{k} \cdots a_{L}^{k} b_{L}^{k}\right\}^{T}$ and $\left\{p_{0}^{k} p_{1}^{k} q_{1}^{k} \cdots p_{L}^{k} q_{L}^{k}\right\}^{T}$, respectively; the first subscript " $j$ " ( $j=0,1,2, \cdots, N$, ) in $\left[\mathbf{U}_{j k}\right]$ and $\left[\mathbf{T}_{j k}\right]$ denotes the index of the $j$ th circle where the collocation point is located and the second subscript " $k$ " $(k=0,1,2, \cdots, N$, ) denotes the index of the $k$ th circle where boundary data $\left\{\varphi_{k}\right\}$ and $\left\{\boldsymbol{\psi}_{k}\right\}$ are specified and $L$ indicates the truncated terms of Fourier series. The coefficient matrix of the linear algebraic system is partitioned into blocks, and each off-diagonal block corresponds to the influence matrices between two different circular holes. The diagonal blocks are the influence matrices due to itself in each individual hole. After uniformly collocating the null-field point along the $k t h$ circular boundary, the submatrix can be written as

$$
\left[\mathbf{K}_{j k}\right]=\left[\begin{array}{cccccc}
K_{j k}^{0 c}\left(\phi_{1}\right) & K_{j k}^{1 c}\left(\phi_{1}\right) & K_{j k}^{1 s}\left(\phi_{1}\right) & \cdots & K_{j k}^{M c}\left(\phi_{1}\right) & K_{j k}^{M s}\left(\phi_{1}\right) \\
K_{j k}^{0 c}\left(\phi_{2}\right) & K_{j k}^{1 c}\left(\phi_{2}\right) & K_{j k}^{1 s}\left(\phi_{2}\right) & \cdots & K_{j k}^{M c}\left(\phi_{2}\right) & K_{j k}^{M s}\left(\phi_{2}\right) \\
K_{j k}^{0 c}\left(\phi_{3}\right) & K_{j k}^{1 c}\left(\phi_{3}\right) & K_{j k}^{1 s}\left(\phi_{3}\right) & \cdots & K_{j k}^{M c}\left(\phi_{3}\right) & K_{j k}^{M s}\left(\phi_{3}\right) \\
\vdots & \vdots & \vdots & \ddots & \vdots & \vdots \\
K_{j k}^{0 c}\left(\phi_{2 L}\right) & K_{j k}^{1 c}\left(\phi_{2 L}\right) & K_{j k}^{1 s}\left(\phi_{2 L}\right) & \cdots & K_{j k}^{M c}\left(\phi_{2 L}\right) & K_{j k}^{M s}\left(\phi_{2 L}\right) \\
K_{j k}^{0 c}\left(\phi_{2 L+1}\right) & K_{j k}^{1 c}\left(\phi_{2 L+1}\right) & K_{j k}^{1 s}\left(\phi_{2 L+1}\right) & \cdots & K_{j k}^{M c}\left(\phi_{2 L+1}\right) & K_{j k}^{M s}\left(\phi_{2 L+1}\right)
\end{array}\right],
$$

where $K$ can be substituted by $U$ or $T$. Although the matrices in Eq.(29) is not sparse, they are diagonally dominant. It is found that the influence coefficient for the higher-order harmonics is smaller. It is noted that the superscript " $0 s$ " in Eq. (29) disappears since $\sin (0 \theta)=0$. The element of $\left[\mathbf{K}_{j k}\right]$ is defined respectively as

$$
\begin{aligned}
& K_{j k}^{n c}\left(\phi_{m}\right)=\int_{B_{k}} K\left(s_{k}, x_{m}\right) \cos \left(n \theta_{k}\right) R_{k} d \theta_{k}, \\
& K_{j k}^{n s}\left(\phi_{m}\right)=\int_{B_{k}} K\left(s_{k}, x_{m}\right) \sin \left(n \theta_{k}\right) R_{k} d \theta_{k},
\end{aligned}
$$

where $n=0,1,2, \cdots, L, m=1,2, \cdots, 2 L+1, k$ is no sum and $\phi_{m}$ is the polar angle of the collocating points $x_{m}$ along the boundary. The physical meaning is that the influence coefficient of $U_{j k}^{n c}\left(\phi_{m}\right)$ in Eq.(30) denotes the response at $x_{m}$ due to the $\cos (n \theta)$ distribution as shown in Figure 3. By rearranging the known and unknown sets, the unknown Fourier coefficients are determined. Equation (20) can be calculated by 


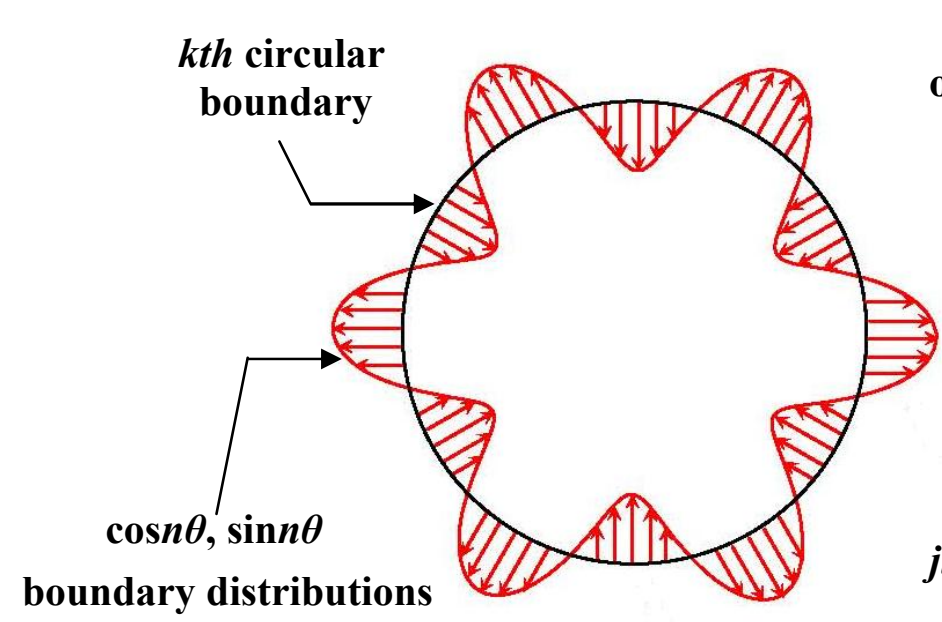

mth collocation point on the jth circular boundary

jth circular boundary

Figure 3: Physical meaning of the influence coefficient $U_{j k}^{n c}\left(\phi_{m}\right)$

employing the orthogonal relations of trigonometric functions in the real computation. Only the finite $L$ terms are used in the summation of Eqs. (23) and (24).

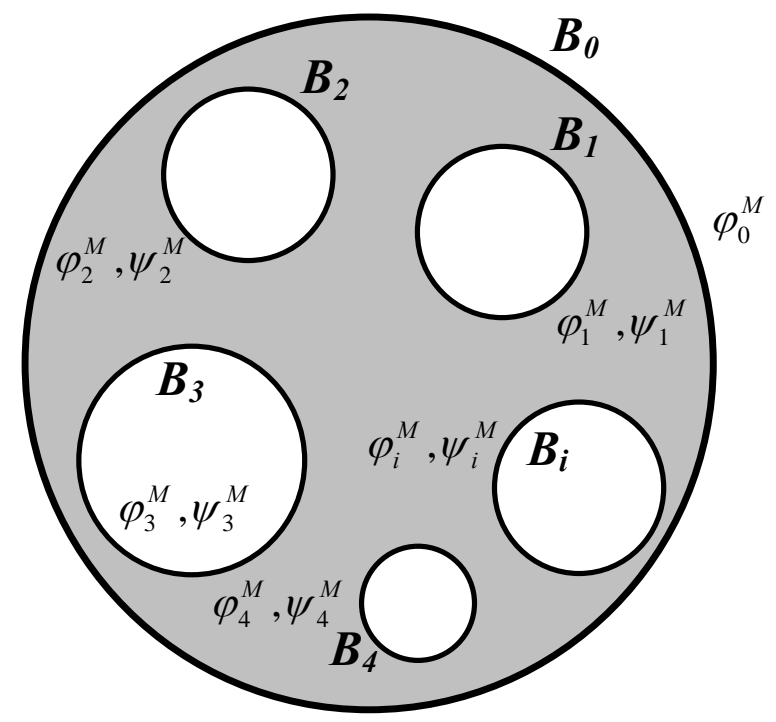

Figure 4(a): Torsion problem of a circular bar with circular holes

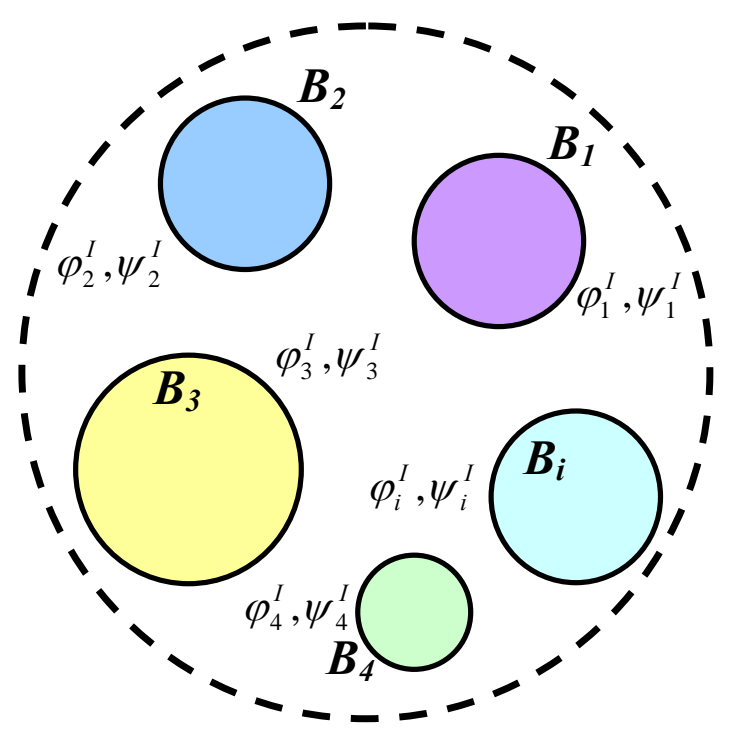

Figure 4(b): Each circular inclusion problem

By using concept of domain decomposition, the problem in Figure 1 can be decomposed into two parts as shown in Figure 4(a) and 4(b). One is the torsion problem of a circular bar with multiple circular holes and the other is a problem of each inclusion. For the torsion problem with circular holes which satisfies the Laplace equation, the linear algebraic system from Eq.(26) can be obtained as

$\left[\begin{array}{ccccccc}\mathbf{T}_{00}^{M} & \mathbf{T}_{01}^{M} & \cdots & \mathbf{T}_{0 N}^{M} & -\mathbf{U}_{01}^{M} & \cdots & -\mathbf{U}_{0 N}^{M} \\ \mathbf{T}_{10}^{M} & \mathbf{T}_{11}^{M} & \cdots & \mathbf{T}_{1 N}^{M} & -\mathbf{U}_{11}^{M} & \cdots & -\mathbf{U}_{1 N}^{M} \\ \vdots & \vdots & \ddots & \vdots & \vdots & \ddots & \vdots \\ \mathbf{T}_{N 0}^{M} & \mathbf{T}_{N 1}^{M} & \cdots & \mathbf{T}_{N N}^{M} & -\mathbf{U}_{N 1}^{M} & \cdots & -\mathbf{U}_{N N}^{M}\end{array}\right]\left\{\begin{array}{c}\varphi_{0}^{M} \\ \varphi_{1}^{M} \\ \vdots \\ \varphi_{N}^{M} \\ \boldsymbol{\psi}_{1}^{M} \\ \vdots \\ \boldsymbol{\psi}_{N}^{M}\end{array}\right\}=\left\{\begin{array}{c}\mathbf{0} \\ \mathbf{0} \\ \vdots \\ \mathbf{0}\end{array}\right\}$. 
For each inclusion, we have

$$
\left[\begin{array}{cccccc}
\mathbf{T}_{11}^{I} & \mathbf{0} & \mathbf{0} & -\mathbf{U}_{11}^{I} & \mathbf{0} & \mathbf{0} \\
\mathbf{0} & \ddots & \mathbf{0} & \mathbf{0} & \ddots & \mathbf{0} \\
\mathbf{0} & \mathbf{0} & \mathbf{T}_{N N}^{I} & \mathbf{0} & \mathbf{0} & -\mathbf{U}_{N N}^{I}
\end{array}\right]\left\{\begin{array}{c}
\varphi_{1}^{I} \\
\vdots \\
\varphi_{N}^{I} \\
\boldsymbol{\psi}_{1}^{I} \\
\vdots \\
\boldsymbol{\psi}_{N}^{I}
\end{array}\right\}=\left\{\begin{array}{c}
\mathbf{0} \\
\vdots \\
\mathbf{0}
\end{array}\right\} .
$$

In order to satisfy the continuity conditions of displacement and equilibrium condition of traction on the interface, we have

$\left\{\varphi_{i}^{M}\right\}-\left\{\varphi_{i}^{I}\right\}=\{\mathbf{0}\}$

$\mu_{0}\left\{\boldsymbol{\psi}_{i}^{M}\right\}-\mu_{i}\left\{\boldsymbol{\psi}_{i}^{I}\right\}=\left\{\mathbf{b}^{i}\right\}$

where $\left\{\mathbf{b}^{i}\right\}$ is

$$
\left\{\mathbf{b}^{i}\right\}=\left\{\begin{array}{c}
\left(\mu_{0}-\mu_{i}\right)\left(-e_{y}^{i} \cos \theta_{1}^{i}+e_{x}^{i} \cos \theta_{1}^{i}\right) \\
\left(\mu_{0}-\mu_{i}\right)\left(-e_{y}^{i} \cos \theta_{2}^{i}+e_{x}^{i} \cos \theta_{2}^{i}\right) \\
\vdots \\
\left(\mu_{0}-\mu_{i}\right)\left(-e_{y}^{i} \cos \theta_{2 M}^{i}+e_{x}^{i} \cos \theta_{2 M}^{i}\right) \\
\left(\mu_{0}-\mu_{i}\right)\left(-e_{y}^{i} \cos \theta_{2 M+1}^{i}+e_{x}^{i} \cos \theta_{2 M+1}^{i}\right)
\end{array}\right\} .
$$

Combining with the above mentioned linear algebraic system of Eqs.(34)-(37), the global linear algebraic equation can be obtained by correctly arranging the Fourier coefficients. After obtaining the Fourier coefficients, the torsional rigidity can be easily determined.

\section{ILLUSTSTRATIVE EXAMPLES AND DISCUSSIONS}

In this section, we revisit the torsion problems with inclusions and/or holes which have been solved by Muskhelishvili [2], Petrov [7] and Tang [8] for demonstrating the validity of present method. The torsional rigidity of each example is calculated after determining the unknown Fourier coefficients.

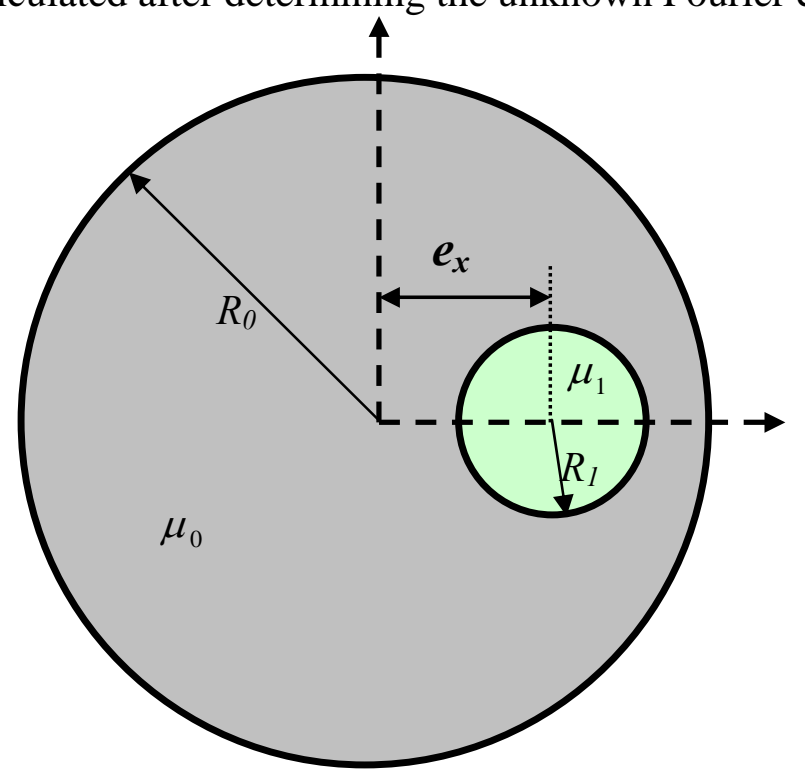

Figure 5: Sketch of an eccentric circular inclusion problem 
1. Case 1: A circular bar with an eccentric inclusion $\mathrm{A}$ circular bar of radius $R_{0}$ with an eccentric circular inclusion of radius $R_{l}$ is shown in Figure 5. The ratio of $R_{l} / R_{0}$ and $e_{x} / R_{0}$ are 0.3 and 0.6 , respectively. The torsional rigidity $G$ of cross section is expressed as

$G=\mu \int_{D}\left(x^{2}+y^{2}\right) d D-\mu \sum_{k=1}^{N} \int_{B_{k}} \varphi \frac{\partial \varphi}{\partial n} d B_{k}$,

$G^{T}=G^{M}+G^{I}$,

where the subscripts " $T$ ", " $M$ " and " $P$ " denote total, matrix and inclusion, respectively. Figure 6 shows the torsional rigidity versus number of Fourier series term when $\mu_{1} / \mu_{0}$ is equal to 0.6. It is found that the solution converges quickly by using only fourteen terms of Fourier series. The results of torsional rigidity for different values of $\mu_{1} / \mu_{0}$ are shown in Table 1 .

Table 1 Torsional rigidity of a circular bar with an eccentric inclusion

\begin{tabular}{|c|c|c|c|}
\hline \multirow{2}{*}{$\mu_{1}$} & \multicolumn{3}{|c|}{$2 G / \pi \mu_{0} R_{0}^{4}$} \\
\hline & Muskhelishvili [2] & Tang [8] & Present method $(M=20)$ \\
\cline { 2 - 4 } & 0.82370 & 0.82377 & 0.82370 \\
\hline 0.2 & 0.89180 & 0.89181 & 0.89180 \\
\hline 0.6 & 0.96246 & 0.96246 & 0.96246 \\
\hline 1.0 & 1.00000 & 1.00000 & 1.00000 \\
\hline 5.0 & 1.10800 & 1.10794 & 1.10800 \\
\hline 20.0 & 1.25224 & 1.25181 & 1.25224 \\
\hline 1000 & 9.19866 & N/A & 9.19866 \\
\hline 10000 & 82.09883 & N/A & 82.09882 \\
\hline 1000000 & 8101.10012 & N/A & 8101.09883 \\
\hline
\end{tabular}

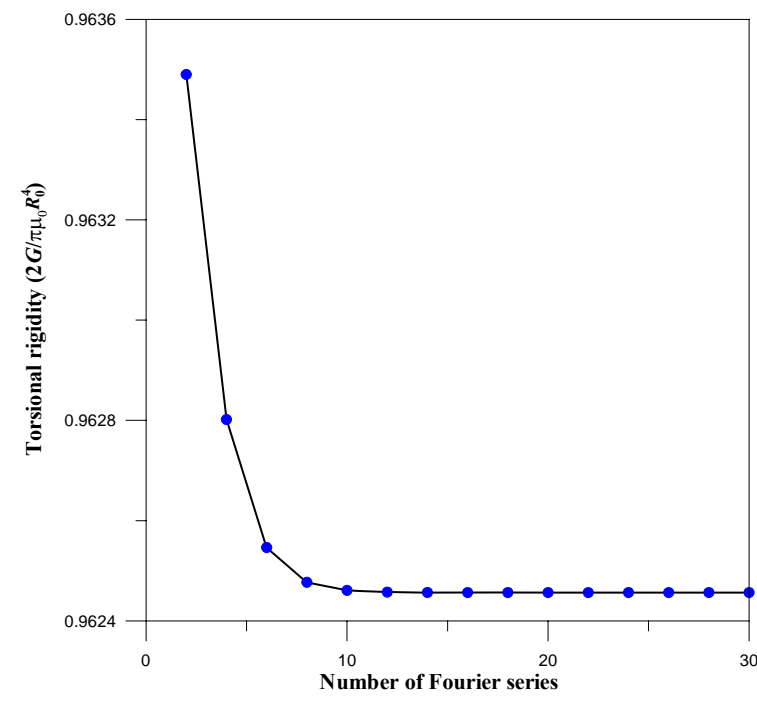

Figure 6: Torsional rigidity versus number of Fourier series terms

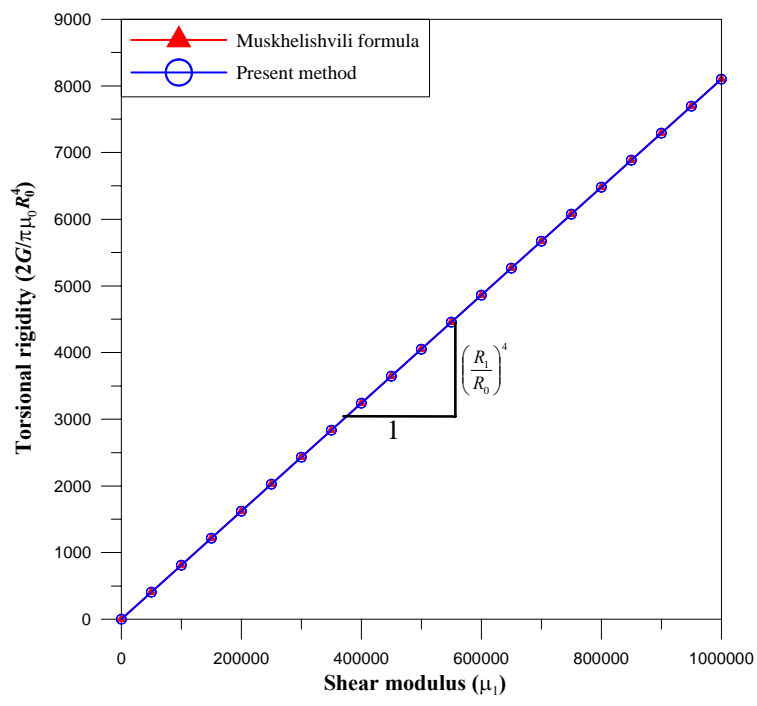

Figure 7: Torsional rigidity versus shear modulus of inclusion

For verifying our results, the Muskhelishvili’s solution is shown below 
$G=\mu_{0} I+\left(\mu_{1}-\mu_{0}\right) I^{\prime}-\frac{\pi R_{1}^{2} e_{x}^{2}\left(\mu_{1}-\mu_{0}\right)^{2}}{\mu_{1}+\mu_{0}}-2 \mu_{0} \pi e_{x}^{2} v \rho_{1}^{2} \sum_{k=1}^{\infty} \frac{\alpha^{k} v^{k}}{\left(1-a^{2} \rho_{1}^{2} \alpha^{k}\right)^{2}}$,

where $I=\frac{\pi R_{0}^{4}}{2}, I^{\prime}=\frac{\pi R_{1}^{4}}{2}+\pi R_{1}^{2} e_{x}^{2}, \quad v=\frac{\left(\mu_{0}-\mu_{1}\right)}{\left(\mu_{0}+\mu_{1}\right)}, a=\frac{e_{x}}{\sqrt{\left(R_{1}^{2}-R_{0}^{2}\right)^{2}-2 e_{x}^{2}\left(R_{1}^{2}+R_{0}^{2}\right)+e_{x}^{4}}}, \alpha=\frac{\rho_{1}^{2}}{\rho_{2}^{2}}$,

$\rho_{1}=\frac{\sqrt{1+4 R_{1}^{2} a^{2}}-1}{2 R_{1} a^{2}}$ and $\rho_{2}=\frac{\sqrt{1+4 R_{0}^{2} a^{2}}-1}{2 R_{2} a^{2}}$. The exact solution of Muskhelishvili and the result of integral formulation by Tang are shown in Table 1 for comparison. The present results matches very well with the exact solution derived by Muskhelishvil and is better than that of Tang. For the rigid inclusion, the torsional rigidity becomes infinity as shown in Table 1. Figure 7 is shown to indicate how shear modulus of inclusion influences the torsional rigidity. It is observed that the slope of torsional rigidity versus $\mu_{1}$ is $\left(R_{1} / R_{0}\right)^{4}$ when the shear modulus of the inclusion becomes large.

2. Case 2: A circular bar with one circular hole (limiting case) The problem is different with the Case 1 by setting zero modulus to simulate the hole. The limiting case is used to check the present formulation. The radius of a circular bar is 1.0 and the radius of the hole is 0.3 . The eccentricity $\left(e_{x}=0.5\right)$ is considered. By using the present method, the shear moduli $\mu_{0}$ and $\mu_{1}$ is chosen 1.0 and 0 , respectively. The exact solution of Muskhelishvili is also calculated by using the exact formula. The results are shown in Table 2. It is found the result of present method matches well with the Muskhelishvili's data and is better than the Petrov's result. However, the Lurje's solution [18] is smaller than those of the Petrov's, Muskhelishvili's and our results. Since three different methods obtain the same result, the formulae of Lurje needs further check.

Table 2 Torsional rigidity of a circular bar with an eccentric hole

\begin{tabular}{|c|c|c|c|}
\hline & \multicolumn{3}{|c|}{$G / \mu_{0} R_{0}^{4}$} \\
\hline Present method & $1.389(34)$ & $1.389(66)$ & $1.389(130)$ \\
\hline Petrov [7] & $1.391(32)$ & $1.390(64)$ & $1.389(130)$ \\
\hline Lurje [18] & & 1.311 & \\
\hline Muskhelishvili [2] & & 1.389 \\
\hline
\end{tabular}

where ( ): number of degrees of freedom

\section{CONCLUSIONS}

Torsion problems with circular inclusions have been successfully solved by using the present formulation. Our solutions match well with the exact solution and other solutions by using the integral formulation. There are only 41 collocation points uniformly distributed on each boundary for more accurate results of torsional rigidity with error less than $1 \%$ after comparing with the exact solution. Although only the eccentric case is used to test the validity of present method, the program is general to deal with arbitrary number, positions, radii and shear moduli of inclusions. Five gains of our approach, (1) free of calculating principal value, (2) exponential convergence, (3) free of boundary-layer effect, (4) meshless method and (5) well-posed model, are obtained. Besides, the BIEs for the domain point or the null-field equation in our formulation can both be used by exactly collocating the point on the real boundary owing to the introduction of the degenerate kernels.

\section{REFERENCES}

1. Ling CB. Torsion of a circular tube with longitudinal circular holes. Quarterly of Applied Mathematics, 1947; 5: 168-181. 
2. Muskhelishvili NI. Some Basic Problems of the Mathematical Theory of Elasticity. Noordhoff, Groningen, 1953.

3. Chen T, Weng IS. Torsion of a circular compound bar with imperfect interface. Journal of Applied Mechanics, ASME, 2001; 68: 955-958.

4. Caulk DA. Analysis of elastic torsion in a bar with circular holes by a special boundary integral method. ASME Journal of Applied Mechanics, 1983; 50: 101-108.

5. Shams-Ahmadi M, Chou SI. Complex variable boundary element method for torsion of composite shafts. International Journal for Numerical Methods in Engineering, 1997; 40: 1165-1179.

6. Ang WT, Kang I. A complex variable boundary element method for elliptic partial differential equations in a multiply-connected region. International Journal of Computer Mathematics, 2000; 75: 515-525.

7. Petrov EP. Analysis of torsion and shear characteristics of beam cross-sections by the boundary element method. The International Journal of BEM Communications, 1997; 8: 239-245.

8. Tang Renji. Torsion Theory of the Cracked Cylinder. Shanghai Jiaotong University Press, Shanghai, China, 1996 (in Chinese).

9. Jin B. A meshless method for the Laplace and biharmonic equations subjected to noisy boundary data. Computer Modeling in Engineering \& Science, 2004; 6(3): 253-262.

10. Sladek V, Sladek J, Tanaka M. Local integral equations and two meshless polynomial interpolations with application to potential problems in non-homogeneous media. Computer Modeling in Engineering \& Science, 2005; 7(1): 69-84.

11. Mogilevskaya SG, Crouch SL. A Galerkin boundary integral method for multiple circular elastic inclusions. International Journal for Numerical Methods in Engineering, 2001; 52: 1069-1106.

12. Crouch SL, Mogilevskaya SG. On the use of Somigliana's formula and Fourier series for elasticity problems with circular boundaries. International Journal for Numerical Methods in Engineering, 2003; 58: 537-578.

13. Bird MD, Steele CR. Separated solution procedure for bending of circular plates with circular holes. ASME Journal of Applied Mechanics, 1991; 59: 398-404.

14. Chen JT, Shen WC, Chen PY. Analysis of circular torsion bar with circular holes using null-field approach. Computer Modelling in Engineering Science, 2006; 12(2): 109-119.

15. Timoshenko SP, Goodier JN. Theory of Elasticity. McGraw-Hill, New York, USA, 1970.

16. Chen JT, Hong HK. Review of dual boundary element methods with emphasis on hypersingular integrals and divergent series. ASME Applied Mechanics Reviews, 1999; 52: 17-33.

17. Chen JT, Chiu YP. On the pseudo-differential operators in the dual boundary integral equations using degenerate kernels and circulants. Engineering Analysis with Boundary Elements, 2002; 26: 41-53.

18. Lurje AI. Theory of Elasticity. Nauka Publisher, Moscow, 1970 (in Russian). 